\title{
On econometric models with rational expectations
}

\section{Laurence Broze and Ariane Szafarz}

Abstract: This chapter is devoted to the analysis of solutions of linear rationalexpectations models. Successively introducing various types of expectations (perfect, naive, adaptive, and rational) in the Muth model, the final reduced forms and the linear stationary solutions are compared. The main solution techniques for rational-expectations models are reviewed on the Cagan model. The "nonuniqueness problem" is also discussed. The reduced form of a very general linear model is given and the linear stationary solutions are parametrically described. The parameters have a simple interpretation and allow for statistical applications. Finally, a generalization to multivariate rational-expectations models is given. If no invertibility conditions are imposed on the structural coefficient matrices, the solution techniques used in the univariate case become insufficient. A method is suggested to obtain the general solution of a multivariate model and to characterize the dimension of the solutions space.

\section{Introduction}

The problem of modeling the mechanism by which economic agents form their expectations is fundamental in macroeconomic theory. In many models, it is essential to include expectations of future variables. However, such expectations are often unobservable. Therefore, assumptions on their formation are needed to complete the specification.

The rational-expectation hypothesis was introduced in a seminal paper by Muth (1961). Several years later the assumption was incorporated in many macromodels (e.g., Sargent and Wallace 1975, 1976; Lucas 1976; Taylor 1979). In these models, expectations are optimal predictions given all the available information. Rational expectations are thus based on an information set that may be chosen by the model builder.

We thank L. P. Hansen for useful comments. 
This chapter is devoted to the analysis of linear rational-expectations models; nonlinear models will be evoked in the conclusion. Since expectations are, in general, unobservable, a full reduction of the model is needed in order to express the endogenous variables in terms of exogenous and lagged variables. It was pointed out by many authors that the nature of rational expectations may lead to multiple solutions, even for simple models. Moreover, different solutions may exhibit very different properties. It is thus important to describe all the solutions.

Various methods were suggested to solve linear rational-expectations models, but only a few of them give the very general solution of the model. Linear stationary solutions described by an infinite moving-average representation (Muth 1961; Taylor 1977; Shiller 1978; Whiteman 1983) or by an autoregressive moving-average (ARMA) representation (Gourieroux, Laffont, and Monfort 1982; Evans and Honkapohja 1986) are often considered. These solutions are obtained through an undetermined coefficients method. Other classes of solutions may be found using undetermined coefficients or a recursive method (McCallum 1976; Blanchard 1979; Blanchard and Kahn 1980; Visco 1981; Wegge 1984; Watson 1985).

For some models, the general solution may be expressed in terms of martingales (Pesaran 1981; Gourieroux et al. 1982; Broze and Szafarz 1984). The limitation of these methods lies in the fact that they cannot be generalized to more sophisticated models. For each model, the problem of its solution must be reconsidered. A global approach is given in Broze, Gourieroux, and Szafarz (1985a). It is based on martingale difference sequences that can be interpreted as revision processes resulting from updating expectations.

In Section 2, we consider the basic model studied by Muth (1961). Successively introducing various types of expectations (perfect, naive, adaptive, and rational), we compare the final reduced forms and the linear stationary solutions.

In Section 3, one of the most popular rational-expectations model, that is, Cagan (1956), is given. It can be viewed as the simplest model exhibiting multiple solutions. For this model, we review the main solution techniques. In particular, the impact of initial and terminal conditions is analyzed. By imposing initial conditions to the endogenous process, one cannot avoid multiplicity. This is the main difference between rationalexpectations models and difference equations. The nonuniqueness problem is the central topic of an abundant literature. Various selection criteria have been considered. Since it is impossible to thoroughly analyze all of them in a single chapter, we will treat, as an example, the case of the minimum-variance conditions given by Taylor (1977). It has to be recog- 
nized, however, that none of these criteria seems totally satisfactory. As an alternative, we suggest a statistical approach based on the parametric description of the linear stationary solutions.

Section 4 is devoted to the study of the solutions of a general model. To this end, we consider the most general linear univariate model with rational expectations and review the results by Broze, Gourieroux, and Szafarz (1985a), giving the general solution and describing parametrically the set of linear stationary solutions. Finally, the comparison with the corresponding perfect-foresight model shows that in some models, rational expectations cannot be perfect. Also the identifiability of the two models is different.

In Section 5, a generalization to multivariate rational-expectations models is given. If no invertibility condition is imposed on the structural coefficient matrices, the solution techniques used in the univariate case become insufficient. Two types of canonical forms of the multivariate models are then introduced. Each leads to an expression of the general solution and has its own utility. The first form gives an algorithm to compute explicit solutions. The second form is used to establish the exact number of arbitrary martingale difference sequences that enter the general solution.

\section{The Muth model}

The model of Muth (1961) is a dynamic version of a classical equilibrium model. The market equations are

$$
\begin{array}{ll}
D_{t}=\gamma p_{t}+\delta+v_{t} & (\gamma<0) \\
S_{t}=\alpha \hat{p}_{t \mid t-1}+\beta+w_{t} & (\alpha>0) \\
D_{t}=S_{t} &
\end{array}
$$

where $D_{t}$ is the demand for good at time $t, S_{t}$ is the supply of good at time $t, p_{t}$ is the market price at time $t, \hat{p}_{t \mid t-1}$ is the expectation of $p_{t}$ held at time $t-1$, and $v_{t}$ and $w_{t}$ are error terms.

The evolution of the equilibrium price is easily deduced from (2.1):

$$
p_{t}=a \hat{p}_{t \mid t-1}+b+u_{t}
$$

where $a=\alpha / \gamma, b=(\beta-\delta) / \gamma, u_{t}=\left(w_{t}-v_{t}\right) / \gamma$. Since an expectation appears in (2.2), an additional assumption on its formation is needed. Nerlove (1958) studied the adaptive case whereas Muth suggested to introduce the rational-expectation hypothesis. In this section, we will compare the results obtained by imposing successively that $\hat{p}_{t \mid t-1}$ is perfect, naive, adaptive, and finally rational. 
2.1 The perfect-foresight version

The strongest assumption on the expectation formation is

$$
\hat{p}_{t \mid t-1}=p_{t}
$$

It says that the price prevailing at time $t$ is known at time $t-1$. Under (2.3), equation ( 2.2 ) becomes

$$
p_{t}=a p_{t}+b+u_{t}
$$

or, equivalently,

$$
p_{t}=\frac{1}{1-a}\left(b+u_{t}\right)
$$

The equilibrium price depends only on the current errors. The model is not really dynamic except for the dynamic effects contained in the term $u_{t}$. At any time, the mean price is the same: $\bar{p}=b /(1-a)$ [of course, with $\left.E\left(v_{t}\right)=E\left(w_{t}\right)=0\right]$.

The naive-expectation version

In this framework, the expectation of $p_{t}$ is given by the past price $p_{t-1}$ :

$$
\hat{p}_{t \mid t-1}=p_{t-1}
$$

Substituting in (2.2) yields

$$
p_{t}=a p_{t-1}+b+u_{t}
$$

The price is thus obtained as a solution of a recursive equation of order 1 . The mean price at time $t, \bar{p}_{t}$, depends on the mean price at time $t-1, \bar{p}_{t-1}$ :

$$
\bar{p}_{t}=a \bar{p}_{t-1}+b
$$

However, if the price is supposed to be stationary, we obtain the same mean $\bar{p}$ as in the perfect-foresight case.

Suppose that the disturbance $u_{t}$ is serially correlated and is, for instance, described by a first-order autoregressive process [AR(1)]:

$$
(1-\phi B) u_{t}=\epsilon_{t} \quad|\phi|<1
$$

where $B$ denotes the lag operator $\left(B u_{t}=u_{t-1}\right)$ and $\epsilon$ is a white noise. When $|a|<1$, the (stationary) price satisfies the relation

$$
(1-\phi B)(1-a B)\left(p_{t}-\bar{p}\right)=\epsilon_{t}
$$

which is an $\operatorname{AR}(2)$ representation. In general, it is easy to show that if $u$ admits an $\operatorname{ARMA}(p, q)$ representation, the process $p-\bar{p}$ has an 
$\operatorname{ARMA}(p+1, q)$ representation (when $|a|<1$ ). When $|a|>1$, recursive equation (2.4) is explosive, and there is no stationary path compatible with it (and such that $p_{t}$ is in the space generated by current and past values of $u_{t}$ ).

\subsection{The adaptive-expectation version}

The expectation of $p_{t}$ made at time $t-1$ is supposed to be a convex combination of the lagged expectation $\hat{p}_{t-1 \mid t-2}$ and the price at time $t-1$ :

$$
\hat{p}_{t \mid t-1}=(1-\lambda) \hat{p}_{t-1 \mid t-2}+\lambda p_{t-1} \quad 0<\lambda \leq 1
$$

From (2.2), we get

$$
p_{t}-(1-\lambda) p_{t-1}=a \lambda p_{t-1}+b \lambda+u_{t}-(1-\lambda) u_{t-1}
$$

and finally,

$$
p_{t}=(a \lambda+1-\lambda) p_{t-1}+b \lambda+u_{t}-(1-\lambda) u_{t-1}
$$

Again, we obtain a recursive equation of order 1 , but this time, a lagged value of $u$ appears as well.

The mean price is now given by

$$
\bar{p}_{t}=(a \lambda+1-\lambda) \bar{p}_{t-1}+b \lambda
$$

The stationarity assumption $\bar{p}_{t}=p$ then ensures that the mean price is equal to the mean price under perfect foresight.

When the disturbance satisfies (2.5), that is, when it has an AR(1) structure, the corresponding stationary solution, for $|a \lambda+1-\lambda|<1$, is

$$
(1-\phi B)[1-(a \lambda+1-\lambda) B]\left(p_{t}-\bar{p}\right)=[1-(1-\lambda) B] \epsilon_{t}
$$

and has thus an $\operatorname{ARMA}(2,1)$ representation. In general, when $u$ is given by an $\operatorname{ARMA}(p, q)$ process and $|a \lambda+1-\lambda|<1, p-\bar{p}$ has an $\operatorname{ARMA}(p+1, q+1)$ representation. When $|a \lambda+1-\lambda|>1$, the recursive equation (2.7) is explosive, and there is no stationary solution.

\section{4}

The rational-expectation version

The expectation is given by the optimal forecast on the basis of information available at time $t-1$. The information set is denoted $I_{t-1}$ :

$$
\hat{p}_{t \mid t-1}=E\left[p_{t} \mid I_{t-1}\right]
$$

Equation (2.2) becomes

$$
p_{t}=a E\left[p_{t} \mid I_{t-1}\right]+b+u_{t}
$$


Such an equation is not a final reduced form of the Muth model with rational expectation since an expectation of the endogenous variable $p_{t}$ is still present. We will call this equation a semireduced form. A (fully) reduced form is an equation in which the current endogenous variable (here $\left.p_{t}\right)$ is expressed in terms of lagged variables $\left(p_{t-1}, p_{t-2}, \ldots\right)$ and exogenous variables (current values, past values, or expectations).

In the simple case of equation (2.9), a fully reduced form is obtained by a projection technique: ${ }^{1}$ the expectation $E\left[p_{t} \mid I_{t-1}\right]$ is computed by taking the conditional expectation of both sides of (2.9):

$$
E\left[p_{t} \mid I_{t-1}\right]=\frac{b}{1-a}+\frac{1}{1-a} E\left[u_{t} \mid I_{t-1}\right]
$$

When the expectation of $p_{t}$ in (2.9) is replaced by this last expression, the final reduced form results:

$$
p_{t}=\frac{b}{1-a}+u_{t}+\frac{a}{1-a} E\left[u_{t} \mid I_{t-1}\right]
$$

No lagged value of price appears in this reduced form, and therefore, $p_{t}$ has always a constant mean equal to $\bar{p}$.

Suppose again that $u_{t}$ verifies (2.5), that is, that it has an AR(1) representation

$$
u_{t}=\phi u_{t-1}+\epsilon_{t} \quad|\phi|<1
$$

Its expectation is then ${ }^{2}$

$$
E\left[u_{t} \mid I_{t-1}\right]=\phi u_{t-1}=u_{t}-\epsilon_{t}
$$

and $(2.10)$ becomes

$$
p_{t}-\bar{p}=u_{t}+\frac{a}{1-a}\left(u_{t}-\epsilon_{t}\right)
$$

or, equivalently,

$$
(1-\phi B)\left(p_{t}-\bar{p}\right)=\left(\frac{1}{1-a}+\frac{a}{1-a}(1-\phi B)\right) \epsilon_{t}
$$

1. This method for solving rational-expectations models has been developed by Visco (1981) in more complex cases. It generalizes results by Aoki and Canzoneri (1979) about models of type

$$
p_{t}=\sum_{k=1}^{r} b_{k} E\left[p_{t} \mid I_{t-k}\right]+\sum_{i=1}^{q} a_{i} p_{t-i}+u_{t}
$$

This model includes no expectations of future variables.

${ }^{2}$ We here assume implicitly that the information set is generated by the $u_{f}$ 's,

$$
I_{t}=\left\{u_{t}, u_{t-1}, \ldots\right\} \mid
$$

To establish reduced-form (2.10), this assumption is not necessary and $I_{t}$ could be larger. 
Table 5.1

\begin{tabular}{llll}
\hline \hline & Reduced form & $\begin{array}{l}\text { Stationarity } \\
\text { condition }\end{array}$ & $\begin{array}{l}\text { Structure of } p \text { if } u \\
\text { is an } \operatorname{ARMA}(p, q)\end{array}$ \\
\hline PF & $p_{t}=\frac{1}{1-a}\left(b+u_{t}\right)$ & ARMA $(p, q)$ \\
NE & $p_{t}=a p_{t-1}+b+u_{t}$ & $|a|<1$ & $\operatorname{ARMA}(p+1, q)$ \\
AE & $p_{t}=(a \lambda+1-\lambda) p_{t-1}$ & $|a \lambda+1-\lambda|<1$ & $\operatorname{ARMA}(p+1, q+1)$ \\
& $\quad+b^{-}+u_{t}-(1-\lambda) u_{t-1}$ & & \\
RE & $p_{t}=\frac{b}{1-a}+u_{t}+\frac{a}{1-a} E\left[u_{t} \mid I_{t-1}\right]$ & - & $\operatorname{ARMA}(p, \max \mid p, q])$ \\
\hline \hline
\end{tabular}

No constraint on the structural coefficient $a$ is needed for stationarity. For any value of $a, p_{t}-\bar{p}$ has an ARMA $(1,1)$ formulation. In general, when $u$ is given by an $\operatorname{ARMA}(p, q)$ process, $p-\bar{p}$ has an $\operatorname{ARMA}(p, \max \{p, q\})$ representation.

\subsection{Comparison of the results}

The results from the previous sections are summarized in Table 5.1. The basic model is

$$
p_{t}=a \hat{p}_{t \mid t-1}+b+u_{t}
$$

The various cases are noted PF (perfect foresight), NE (naive expectation), AE (adaptive expectation), and RE (rational expectation). The stationarity domains can be ordered. The smallest one corresponds to naive expectation. Then comes the adaptive expectation. Finally, the perfectforesight and rational-expectations cases impose no restriction on the parameters. "Quality" in forecasting thus seems to provide larger stability domains in terms of structural parameters. Notice that from this point of view, there is no distinction between perfect and rational expectations.

Finally, it should be noted that, in all cases, the stationary solution has a mean equal to $\bar{p}=b /(1-a)$, which is the mean price (without any stationarity assumption) for the perfect-foresight case as well as for the rational-expectation case.

\subsection{Conditions for identical predictions}

We have shown that under an ARMA assumption on the error term, the stationarity condition and the ARMA path for prices are not the same for 
all prediction schemes. One can inquire whether there exists a structure for $u$ such that the rational expectation coincides with another type of expectation. We will successively impose the coincidence of the rational expectation with the PF, NE, and $\mathrm{AE}$.

\subsection{1 $E\left[p_{t} \mid I_{t-1}\right]=p_{t}$}

This condition is equivalent to requiring no prediction error in $p_{t}$. However, (2.10) shows that there is a one-to-one relationship between the error on $p_{t}$ and the error on $u_{t}$ :

$$
p_{t}-E\left[p_{t} \mid I_{t-1}\right]=u_{t}-E\left[u_{t} \mid I_{t-1}\right]
$$

Therefore, (2.13) is verified if and only if $u_{t}=E\left[u_{t} \mid I_{t-1}\right]$, that is, if the disturbance is predetermined.

$$
\text { 2.6.2 } E\left[p_{t} \mid I_{t-1}\right]=p_{t-1}
$$

From (2.10), we obtain

$$
E\left[p_{t} \mid I_{t-1}\right]-p_{t-1}=\frac{1}{1-a} E\left[u_{t} \mid I_{t-1}\right]-u_{t-1}-\frac{a}{1-a} E\left[u_{t-1} \mid I_{t-2}\right]
$$

Condition (2.14) becomes

$$
E\left[u_{t} \mid I_{t-1}\right]-(1-a) u_{t-1}-a E\left[u_{t-1} \mid I_{t-2}\right]=0
$$

Conditioning on $I_{t-2}$ yields

$$
E\left[u_{t} \mid I_{t-2}\right]=E\left[u_{t-1} \mid I_{t-2}\right]
$$

A necessary condition is that, at time $t-2$, the expected values of $u_{t}$ and $u_{t-1}$ are equal.

If $u$ has an infinite moving-average representation,

$$
u_{t}=\epsilon_{t}+\theta_{1} \epsilon_{t-1}+\theta_{2} \epsilon_{t-2}+\cdots
$$

condition (2.15) is equivalent to

$$
\theta_{1}=\theta_{2}=\cdots=1-a
$$

This is incompatible with the stationarity restriction on $u_{t}: \sum_{i=1}^{\infty} \theta_{i}^{2}<\infty$. Nevertheless, condition $(2.15)$ can be satisfied. For instance, when $u$ is time invariant,

$$
u_{t}=u_{t-1}=u_{t-2}=\cdots=u
$$

it is rational to use $p_{t-1}$ as a prediction of $p_{t}$. 


$$
\text { 2.6.3 } E\left[p_{t} \mid I_{t-1}\right]=(1-\lambda) E\left[p_{t-1} \mid I_{t-2}\right]+\lambda p_{t-1} \quad 0<\lambda \leq 1
$$

From (2.9), we obtain

$$
E\left[p_{t} \mid I_{t-1}\right]=\frac{b}{1-a}+\frac{1}{1-a} E\left[u_{t} \mid I_{t-1}\right]
$$

and (2.16) becomes

$$
\frac{1}{1-a} E\left[u_{t} \mid I_{t-1}\right]=\frac{1-\lambda}{1-a} E\left[u_{t-1} \mid I_{t-2}\right]+\lambda u_{t-1}+\frac{\lambda a}{1-a} E\left[u_{t-1} \mid I_{t-2}\right]
$$

or

$$
\frac{1}{1-a} E\left[u_{t} \mid I_{t-1}\right]=\frac{1-\lambda+a \lambda}{1-a} E\left[u_{t-1} \mid I_{t-2}\right]+\lambda u_{t-1}
$$

Thus, the stochastic process $u$ verifies an equation including two different rational expectations. We will show further that this equation has an infinity of solutions for any value of $\lambda$.

\section{3}

\section{The Cagan model}

Cagan's (1956) hyperinflation model describes a market of money where inflationary expectations play a central role. The equations of the model are:

$$
m_{t}^{d}-p_{t}=\alpha\left(\hat{p}_{t+1 \mid t}-p_{t}\right) \quad m_{t}^{s}=z_{t} \quad m_{t}^{d}=m_{t}^{s}
$$

with all variables denoting logarithms. The first equation gives the realmoney demand as a function of the expected rate of inflation. The structural coefficient $\alpha$ is often taken to be negative. Blanchard (1979) noted, however, that the sign of $\alpha$ depends on the elasticity of substitution between consumption in the two successive periods. He does not exclude the case $\alpha>0$, which occurs when the elasticity is larger than 1 . The second equation states that money supply is exogenous. The third is the equilibrium condition.

The evolution of the equilibrium price is given by

$$
p_{t}=a \hat{p}_{t+1 \mid t}+u_{t}
$$

where $a=\alpha /(\alpha-1)$ and $u_{t}=-z_{t} /(\alpha-1)$. The stochastic process $u$ is exogenous since it is obtained from $z$.

Model (3.2) is different from (2.2): The expectation $\hat{p}_{t \mid t-1}$ has been replaced by $\hat{p}_{t+1 \mid r}$. More precisely, two modifications have been introduced. First, there is an expectation of a future endogenous variable $p_{t+1}$. Second, this expectation is formed at time $t$, that is, simultaneously with 
the realization of $p_{t}$. Of course, under the perfect-foresight assumption, the second modification has no impact. However, when expectations are assumed rational, the reduced form is influenced by both changes.

Under rational expectations, model (3.2) becomes

$$
p_{t}=a E\left[p_{t+1} \mid I_{t}\right]+u_{t}
$$

First, notice that the projection technique used for solving the Muth model does not apply for solving (3.3). Taking the conditional expectation of both sides of (3.3) with respect to $I_{t}$, one gets only the same equation.

Moreover, it has now clearly been established that such a model has an infinity of solutions. The nonuniqueness problem was pointed out by many authors. Most of them exhibited particular solutions. Others gave the general solution and sometimes suggested selection criteria.

The forward-backward approach

A natural method for solving (3.3) is given by successive substitutions (Blanchard 1979). The forward-looking procedure leads to

$$
\begin{aligned}
p_{t} & =a E\left[p_{t+1} \mid I_{t}\right]+u_{t} \\
& =a^{2} E\left[p_{t+1} \mid I_{t}\right]+a E\left[u_{t+1} \mid I_{t}\right]+u_{t} \\
& \vdots \\
& =u_{t}+a E\left[u_{t+1} \mid I_{t}\right]+a^{2} E\left[u_{t+2} \mid I_{t}\right]+\cdots+a^{k} E\left[u_{t+k} \mid I_{t}\right]+\cdots
\end{aligned}
$$

The infinite sum does not always converge. When it does, it provides a solution for model (3.3). For instance, when $|a|>1$ and $u$ is a stationary process, the so-called forward solution does exist.

The backward-looking procedure does not work so simply because one has to "extract" the variable $p_{t+1}$ out of the expectation $E\left[p_{t+1} \mid I_{t}\right]$. One way of doing this is provided by the perfect-foresight assumption:

$$
E\left[p_{t+1} \mid I_{t}\right]=p_{t+1}
$$

Under this additional condition, rational-expectations model (3.3) becomes a difference equation of order 1 :

$$
p_{t}=a p_{t+1}+u_{t}
$$

Recursive substitutions lead to

$$
\begin{aligned}
p_{t} & =\frac{1}{a} p_{t-1}-\frac{1}{a} u_{t-1} \\
& =\frac{1}{a^{2}} p_{t-2}-\frac{1}{a^{2}} u_{t-2}-\frac{1}{a} u_{t-1} \\
& \vdots \\
& =\frac{u_{t-1}}{a}-\frac{u_{t-2}}{a^{2}}-\cdots-\frac{u_{t-k}}{a^{k}}-\cdots
\end{aligned}
$$


When this infinite sum converges, it gives a particular solution of the $\mathrm{Ca}$ gan model. For instance, this "backward solution" or "perfect-foresight solution" exists when $|a|>1$ and $u$ is stationary.

Comparing the forward and the backward approaches, one observes the asymmetry between the past and the future in rational-expectations models. Whereas a terminal condition (see Chow 1980) does fix one solution (the forward development is then finite), an initial condition may only be imposed at this stage on a perfect-foresight solution. Moreover, the forward solution is also the special solution satisfying the transversality condition in the permanent-income model of consumption studied, for instance, by Hansen and Sargent $(1980,1982)$.

\subsection{Undetermined coefficients}

The undetermined-coefficients method is based on a "guess" regarding the form of the solution(s), followed by an identification of undetermined coefficients. This method was largely used in order to obtain ARMA or infinite MA solutions (Muth 1961; Taylor 1977; Gourieroux et al. 1982; McCallum 1983; Whiteman 1983; Evans and Honkapohja 1986; Evans 1985).

The exogenous process $u$ is supposed to have an infinite MA representation that is invertible and based on an independent white noise denoted $\epsilon$ :

$$
u_{t}=\epsilon_{t}+\alpha_{1} \epsilon_{t-1}+\cdots+\alpha_{k} \epsilon_{t-k}+\cdots=A(B) \epsilon_{t} \quad \sum_{k=0}^{\infty} \alpha_{K}^{2}<\infty
$$

The solutions of model (3.3) admitting the same structure (henceforth the linear stationary solutions) may be obtained by the undetermined-coefficients method. The "guess" settles the expression:

$$
p_{t}=\psi_{0} \epsilon_{t}+\cdots+\psi_{k} \epsilon_{t-k}+\cdots=\Psi(B) \epsilon_{t} \quad \sum_{k=0}^{\infty} \psi_{K}^{2}<\infty
$$

It follows that

$$
E\left[p_{t+1} \mid I_{t}\right]=\frac{\Psi(B)-\psi_{0}}{B} \epsilon_{t}
$$

Replacing $p_{t}, E\left[p_{t+1} \mid I_{t}\right]$, and $u_{t}$ by their expressions in model (3.3) yields

$$
\Psi(B)=a \frac{\Psi(B)-\psi_{0}}{B}+A(B)
$$

or

$$
\Psi(B)=\frac{B A(B)-a \psi_{0}}{B-a}
$$

The coefficients $\psi_{k}$ do not necessarily satisfy the condition $\sum_{k=0}^{\infty} \psi_{k}^{2}<\infty$. Two cases must be distinguished. 
(i) If $|a|<1$, one must impose that the structural parameter $a$ is a root of the numerator, that is, that $\psi_{0}=A(a)$. The linear stationary solution is then unique. It is given by

$$
p_{t}=\frac{B A(B)-a A(a)}{B-a} \epsilon_{t}
$$

In fact, this solution is the forward solution of the model.

(ii) If $|a|>1$, any value of $\psi_{0}$ leads to a linear stationary solution. There is a multiplicity of such solutions parameterized by $\psi_{0}$ :

$p_{t}=\frac{B A(B)-a \psi_{0}}{B-a} \epsilon_{t} \quad \psi_{0} \in \mathbb{R}$

After a change of the parameter $\psi_{0}$, one can see that these solutions are combinations of the forward solution

$$
p_{i}^{1}=\frac{B A(B)-a A(a)}{B-a} \epsilon_{t}
$$

and the backward solution

$$
p_{i}^{2}=\frac{B A(B)}{B-a} \epsilon_{t}
$$

\subsection{General solution using the homogenous equation}

The approach followed by Gourieroux et al. (1982) is inspired by the solution technique used for linear recursive equations. First, the general solution of model (3.3) is decomposed as

$$
p_{t}=p_{t}^{0}+p_{i}^{*}
$$

where $p^{0}$ is a particular solution of the model and $p^{*}$ is the general solution of the homogeneous equation obtained by replacing the exogenous process by 0 :

$$
p_{i}^{*}=a E\left[p_{i+1}^{*} \mid I_{t}\right]
$$

The solutions of the last equation are obtained by noting that the process $M_{t}=a^{t} p_{t}^{*}$ is such that the rational expectation of $M_{t+1}$ coincides with the last value $M_{t}$ :

$$
E\left[M_{t+1} \mid I_{t}\right]=M_{t}
$$

Such a process is called a martingale. Finally, the general solution of model (3.3) is given by

$$
p_{t}=p_{t}^{0}+\frac{1}{a^{t}} M_{t}
$$

where $p^{0}$ is a particular solution, and $M$ is an arbitrary martingale. 
According to the value of the structural parameter $a$, one can choose as particular solution either the forward solution $(|a|<1)$ or the backward solution $(|a|>1)$. The model has an infinity of solutions described by an arbitrary martingale.

We again observe the asymmetry between the past and the future: $\mathrm{A}$ martingale is fixed by a terminal condition $\left(M_{t}=E\left[M_{T} \mid I_{t}\right]\right)$ but not by an initial condition. The argument here is stronger than before since it holds for all solutions (and not only for the perfect-foresight solution). For this reason, a rational-expectations model is very different from a deterministic difference equation.

\subsection{General solution using revision processes}

The method (Broze, Gourieroux, and Szafarz 1985a) is a kind of backward approach without the perfect-foresight assumption. The variable $p_{t+1}$ is "extracted" out of the expectation $E\left[p_{t+1} \mid I_{t}\right]$ by means of the prediction error:

$$
\epsilon_{t}^{0}=p_{t}-E\left[p_{t} \mid I_{t-1}\right]
$$

The variable $\epsilon_{t}^{0}$ is a function of the current information $I_{t}$ and is orthogonal to past information: $E\left[\epsilon_{t}^{0} \mid I_{t-1}\right]=0$. A process having these two properties is called a martingale difference sequence.

Replacing in the original model the expectation $E\left[p_{t+1} \mid I_{t}\right]$ by the difference between the realization and the prediction error, we see that any solution of the model has to satisfy the equation

or

$$
p_{t}=a\left(p_{t+1}-\epsilon_{t+1}^{0}\right)+u_{t}
$$

$$
p_{t}=\frac{1}{a} p_{t-1}-\frac{1}{a} u_{t-1}+\epsilon_{t}^{0}
$$

Conversely, any solution of the last equation does satisfy model (3.3). Equation (3.4) is thus a fully reduced form of Cagan's model that involves an arbitrary martingale difference sequence. Furthermore, this expression of the solutions is useful to distinguish two causes of multiplicity:

(i) The dynamic features of the model lead to a first-order linear difference equation giving $p_{t}$ as a function of $p_{t-1}$. This "classical" multiplicity may be avoided by giving an initial value $p_{0}$.

(ii) The "specific" multiplicity comes out the possible choice of $\epsilon_{t}^{0}$. Intuitively, it may be explained as follows: At time $t$, the equilibrium price, $p_{t}$, and the expectation, $E\left[p_{t+1} \mid I_{t}\right]$, are known. However, the prediction error, $p_{t+1}-E\left[p_{t+1} \mid I_{t}\right]$, leading to the next equilibrium price, $p_{t+1}$, is arbitrary (as a martingale difference sequence). For Cagan's model, the rational-expectation hy- 
pothesis is not sufficient to obtain a unique path for the equilibrium price, even with a fixed initial value.

The method using revision processes looks well adapted for determination of the linear stationary solutions. Suppose that the exogenous process has the representation $u_{t}=A(B) \epsilon_{t}$ and consider the solutions having the same form: $p_{t}=\Psi(B) \epsilon_{t}$. The prediction error, $\epsilon_{t}^{0}=p_{t}-E\left[p_{t} \mid I_{t-1}\right]$, is necessarily proportional to the white noise $\epsilon_{t}$ :

$$
\epsilon_{t}^{0}=\psi_{0} \epsilon_{t}
$$

Therefore, the linear stationary solutions must fulfill the equation

$$
p_{t}=\frac{1}{a} p_{t-1}-\frac{1}{a} u_{t-1}+\psi_{0} \epsilon_{t}=\frac{B A(B)-a \psi_{0}}{B-a} \epsilon_{t}
$$

Obviously, two cases have to be distinguished according to the value of $|a|$ relative to 1 (see Section 3.2 ). The extra parameter $\psi_{0}$ describing the linear stationary solutions has a simple statistical interpretation that may be used for further estimation. This parameter is the regression coefficient of the prediction error on $p\left(p_{t}-E\left[p_{t} \mid I_{t-1}\right]\right)$ when regressed against the prediction error on $u\left(u_{t}-E\left[u_{t} \mid I_{t-1}\right]\right)$.

Nonlinear stationary solutions may be considered. Assume, for simplicity, that $u_{t}=\epsilon_{t}$ is an independent white noise and $|a|>1$. Given any function $f$ for which $E f\left(\epsilon_{t}\right)$ exists, the process $\epsilon_{t}^{0}=f\left(\epsilon_{t}\right)-E f\left(\epsilon_{t}\right)$ is a martingale difference sequence. The process defined by

$$
p_{t}=\left(1-\frac{B}{a}\right)^{-1}\left(-\frac{1}{a} \epsilon_{t}+f\left(\epsilon_{t}\right)-E f\left(\epsilon_{t}\right)\right)
$$

is then a solution of the model. It is a stationary process that is generally nonlinear with respect to $\epsilon$. There exists a large number of stationary solutions different from those admitting an ARMA representation based on $\epsilon$. Moreover, since the autocorrelation function of $(-1 / a) \epsilon_{t-1}+f\left(\epsilon_{t}\right)-$ $E f\left(\epsilon_{t}\right)$ vanishes after the second order, there exists a white noise $\eta_{t}$ such that

$$
-(1 / a) \epsilon_{t-1}+f\left(\epsilon_{t}\right)-E f\left(\epsilon_{t}\right)=\eta_{t}-\theta \eta_{t-1}
$$

All solutions given by $(3.6)$ have an $\operatorname{ARMA}(1,1)$ representation: They are linear with respect to white noise $\eta$. This white noise is not the innovation of the exogenous process. In general, it is not independent but only nonautocorrelated.

\subsection{Minimum-variance solution}

The previous results imply that a problem of nonuniqueness appears for rational-expectations models when expectations of future endogenous 
variables enter those models. This problem might be quite embarrassing for econometric modeling.

Faced with the problem, several authors have suggested adding conditions in order to select specific solution(s). Those approaches are based on a priori restrictions that complete the specification of the model. As shown above, stationarity does not always lead to uniqueness. Other criteria have also been considered: boundedness in mean, predictive power, and so on (see Gourieroux et al. 1982). However, none of these was singled out since none does provide uniqueness for any value of the structural coefficients and for any type of exogenous process. Another way of selecting solutions is given by the study of learning processes (Bray 1981; Bray and Savin 1984; Fourgeaud, Gourieroux, and Pradel 1986; Bowden 1984). Finally, it is also possible to introduce an optimization criterion based on a given objective function. Among those quadratic criteria, Taylor (1977) has suggested the minimum-variance condition. In this section, we will further analyze this type of approach. For simplicity, Taylor's criterion will be considered. However, results could be generalized to other quadratic optimization problems. For instance, Chow (1980) and Whiteman (1985) related the choice of a solution to a problem of optimal control.

Although stationarity does not, by itself, solve the nonuniqueness problem, it is a quite reasonable restriction, at least if one is concerned with "stable" solutions. Taylor (1977) has added to stationarity the minimumvariance condition, which also conveys the idea of stability. For a particular model, he showed that the linear stationary solution of minimum variance is unique: It is associated with a specific value of the parameter describing the linear stationary solutions. The same criterion was used by Gourieroux et al. (1982) in their analysis of Cagan's model and a similar result was obtained. Notice that this approach is entirely based on a priori restrictions on the set of solutions. Further, we will show that an a posteriori statistical procedure may also be considered.

In fact, the apparently satisfactory application of the minimum-variance condition has strong limitations, especially when the linearity and stationarity assumptions are avoided. Recall that the work by Taylor and Gourieroux et al. about the minimum-variance solution did only concern linear stationary solutions. Since there exists a large number of other solutions (even stationary ones), it is quite natural to enquire whether the minimality property might be generalized to all solutions. The answer to this problem is related to the structure of the model. Hence, we will first consider Taylor's model and then Cagan's model.

\subsubsection{Taylor's model}

The structural form of Taylor's (1977) model contains four equations: 


$$
\begin{aligned}
& y_{t}=-\gamma_{1}\left(i_{t}-E\left[p_{t+1} \mid I_{t-1}\right]+E\left[p_{t} \mid I_{t-1}\right]\right)+\gamma_{2}\left(m_{t}-p_{t}\right)+\epsilon_{1 t} \\
& y_{t}=\phi_{0}+\phi_{1}\left(m_{t}-p_{t}\right)+\epsilon_{2 t} \\
& m_{t}=y_{t}+p_{t}-\alpha_{1} i_{t}+\alpha_{2}\left(m_{t}-p_{t}\right)-\epsilon_{3 t} \\
& m_{t}=m
\end{aligned}
$$

All variables are expressed in logarithms. The first equation gives the demand for goods. It depends negatively on the real rate of interest and positively on real-money balances. The second equation gives the supply. It depends on the level of real-money balances. The third equation gives the money demand as a function of the nominal income, the nominal rate of interest, and the level of real-money balance. The last equation gives a constant money supply.

The evolution of the equilibrium price is then given by the following rational-expectations model:

$$
p_{t}=\frac{1}{\delta_{1}} E\left[p_{t+1} \mid I_{t-1}\right]-\frac{1}{\delta_{1}} E\left[p_{t} \mid I_{t-1}\right]-\frac{\delta_{0}}{\delta_{1}}-\frac{1}{\delta_{1}} \epsilon_{t}
$$

where $\epsilon$ is an independent white noise with variance $\sigma^{2}$. Taylor shows that the particular solution

$$
p_{t}^{0}=-\frac{\delta_{0}}{\delta_{1}}+\frac{1}{\delta_{1}} \epsilon_{t}
$$

has minimum variance in the set of linear stationary solution,

$$
p_{t}=\bar{p}+\theta(B) \epsilon_{t}=\bar{p}+\theta_{0} \epsilon_{t}+\theta_{1} \epsilon_{t+1}+\cdots
$$

that is, in the set of moving-average solutions based on the white noise $\epsilon$.

In fact, it turns out that $p_{t}^{0}$ verifies the minimum-variance condition in the set of all solutions. Using (3.6) and taking the variance of both sides,

$$
\begin{aligned}
V\left(p_{t}\right) & =\left(1 / \delta_{1}\right)^{2} V\left(E\left[p_{t+1} \mid I_{t-1}\right]-E\left[p_{t} \mid I_{t-1}\right]\right)+\left(1 / \delta_{1}\right)^{2} \sigma^{2} \\
& \geq\left(1 / \delta_{1}\right)^{2} \sigma^{2} \\
& =V\left(p_{t}^{0}\right)
\end{aligned}
$$

\subsubsection{Cagan's model}

Consider the Cagan model with rational expectation:

$$
p_{t}=a E\left[p_{t+1} \mid I_{t}\right]+u_{t}
$$

where the exogenous process $u$ is stationary with an infinite moving-average representation: 


$$
u_{t}=\Theta(B) \epsilon_{t}=\epsilon_{t}+\theta_{1} \epsilon_{t-1}+\cdots
$$

where $\epsilon$ is an independent white noise, with variance $\sigma^{2}$, which can be interpreted as the innovation of the process $u$. Gourieroux et al. (1982) have solved for the minimum-variance solution in the set of linear stationary solutions. Contrary to the model of Taylor, it is impossible to extend this property to the set of all solutions of the Cagan model.

Theorem 3.1. There exists no minimum-variance solution in the set of all solutions of the Cagan model.

Proof. See Appendix A.

Summarized, the proof of Theorem 3.1 shows that a solution can be constructed so that it has a zero variance for any given date. It is not possible, however, for the same solution to be minimum variance at all dates. Therefore, a solution that is minimum variance at all dates does not exist.

Since there exists no minimum variance in the set of all solutions, we now consider the set of solutions such that the bidimensional process $\left(p_{t}, u_{t}\right)$ is stationary. Obviously, we study only the nontrivial case $|a|>1$ such that the set contains more than one element.

Theorem 3.2. There exists a minimum-variance solution in the set of stationary solutions of the Cagan model. It is the minimum-variance solution (determined by Gourieroux et al. 1982) in the set of linear stationary solutions.

Proof. See Appendix B.

Gourieroux et al. have exhibited a minimum-variance solution in the set of linear stationary solutions of the Cagan model. We have shown that this solution is also minimum variance in the set of all stationary solutions, whether linear or nonlinear. This extension is important since, in fact, there exists a large number of nonlinear stationary solutions [see, e.g., formula (3.6)].

In conclusion, the minimum-variance condition may be used for some rational-expectations models, like Taylor's model, to select a unique solution among the set of all solutions. However, for some other cases, like the Cagan model, it may select no solution. In this case, the minimumvariance condition may be considered as a complement to the stationarity condition since it is well known that this last condition alone does not generally give a unique solution. 


\subsection{A statistical approach}

Solving the nonuniqueness problem by means of selection criteria is often criticized as "ad hoc procedures." Moreover, their specific failures make selection criteria highly unsatisfactory.

Another approach is suggested by a parameterization of the linear stationary solutions. It was shown that the parameter $\psi_{0}$ appearing in expression (3.5) may be interpreted as the regression coefficient of the prediction error on the current price over the prediction error on the current exogenous variable. Admitting a priori that the observations $p_{1}, \ldots, p_{T}$ correspond to a moving-average solution, $\psi_{0}$ may be estimated, for instance, by the following method:

(a) a consistent approximation, $\tilde{\tilde{p}}_{t+1}$ of $E\left[p_{t+1} \mid I_{t}\right]$ is deduced from the autoregression of $p_{t+1}$ on $p_{t}, p_{t-1}, \ldots$ :

$$
{ }_{t} \tilde{\tilde{p}}_{t+1}=\sum_{k=0}^{k_{t}} \downarrow_{k, T} p_{t-k}
$$

(b) If the structural coefficient $a$ is identifiable and consistently estimable by $\hat{a}_{T}$, it is possible to compute the residuals:

$\tilde{u}_{t}=p_{t}-\hat{a}_{T t} \tilde{\bar{p}}_{t+1}$

They are consistent approximations of the variables $u_{t}$.

(c) The expectation $E\left[u_{t} \mid I_{t-1}\right]$ is then approached by means of an autoregression of $\tilde{\tilde{u}}_{t}$ on the lagged values $\tilde{\tilde{u}}_{t-1}, \tilde{\tilde{u}}_{t-2}, \ldots$.

(d) Calling $t_{t-1} \tilde{u}_{t}$ this last approximation, the parameter $\psi_{0}$ may be consistently estimated by regressing $p_{t}-t_{t-1} \tilde{\tilde{p}}_{t}$ on $\tilde{\tilde{u}}_{t}-\psi_{t-1} \tilde{\tilde{u}}_{t}$.

This procedure shows that the (asymptotic) identification of the structural coefficient $a$ implies that the parameter $\psi_{0}$ is also identified. Notice that this method still has to be applied with a priori (classical) restrictions (linearity and stationarity). It is, however, an a posteriori technique to single out one solution in a large class of parameterized solutions. Moreover, it emphasizes that the rational-expectation hypothesis does not necessarily yield empirical undeterminacy.

The general model

The most general form of a unidimensional linear rational-expectations model is

$$
y_{t}=\sum_{k=0}^{K} \sum_{h=1}^{H} a_{k h} E\left[y_{t+h-k} \mid I_{t-k}\right]+\sum_{k=1}^{K} a_{k 0} y_{t-k}+u_{t}
$$


The current endogenous variable $y_{t}$ depends on its lagged values $y_{t-1}$, $y_{t-2}, \ldots$, on past and current expectations $E\left[y_{t+h-k} \mid I_{t-k}\right]$ and on a variable $u_{t}$ summarizing all exogenous influences as well as the disturbances at time $t$.

The information available at time $t$ is denoted $I_{t}$. It increases with $t$ and contains at least the values of the exogenous process:

$$
I_{t} \supset\left\{u_{t}, u_{t-1}, \ldots\right\}
$$

The set $I$, may, however, be larger and include other variables such as "sunspots."

In model (4.1), the index $h$ denotes the horizon of each expectation, and $t-k$ is the period at which the expectation is formed. Among the coefficients $a_{k H}, k=0, \ldots, K$ (respectively $a_{K h}, h=0, \ldots, H$ ), one at least is not zero, so that $H$ and $K$ are uniquely defined. This convention allows for the interpretation of $H$ as the maximum horizon appearing in the model.

The model could include current and past expectations about future values as well as past expectations about past values. For obvious reasons, expectations using future observations are excluded; the index $k$ takes only positive values. Therefore, $y_{t}$ is a function of the variables appearing in $I_{t}$. The lagged endogenous variables may be seen as special expectations:

$$
y_{t-k}=E\left[y_{t-k} \mid I_{t-k}\right]
$$

They may be incorporated in the model (with $a_{00}=0$ )

$$
y_{t}=\sum_{k=0}^{K} \sum_{h=0}^{H} a_{k h} E\left[y_{t+h-k} \mid I_{t-k}\right]+u_{t}
$$

\subsection{Reduced form of the model}

A reduced form of model (4.1) is given in Broze, Gourieroux, and Szafarz (1985a) using martingale difference sequences. For this purpose, the model can be reformulated as follows, setting $i=h-k$.

$$
y_{t}=\sum_{(k, i) \in \mathcal{S}} a_{k, i+k} E\left[y_{t+i} \mid I_{t-k}\right]+u_{t}
$$

where $\mathscr{J}=\{(k, h-k), h \in\{0, \ldots, H\}, k \in\{0, \ldots, K\}]$. The new index $i$ refers to the expected variable $y_{t+i}$ regardless of the period at which the expectation is formed. The maximal value of $i$, denoted $J_{1}$, gives the number of arbitrary martingale difference sequences needed to describe all solutions. The fundamental results may be summarized as follows ${ }^{3}$

${ }^{3}$ Strictly speaking, this result holds under nullity constraints on the structural parameters (i.e., it is generic in the space of the coefficients nonsubject to nullity constraints). If this 
(i) Model (4.1) has a reduced form entailing $J_{1}\left(0 \leq J_{1} \leq H\right)$ arbitrary martingale difference sequences.

(ii) Its reduced form is given by

$$
\sum_{i=J_{0}}^{J_{1}} a_{i}^{*} y_{t+i}=\sum_{j=H-J_{1}}^{H-1} \sum_{k=k_{j}}^{K_{j}} c_{j k} \epsilon_{i+J_{1}-k}^{j}+g_{i}(u)
$$

where the coefficients $a_{i}^{*}$ and $c_{j k}$ are combinations of the structural coefficients $a_{k h ;} ; g_{t}(u)$ is a known function of the exogenous process; and $\epsilon^{H-J_{1}}, \ldots, \epsilon^{H-1}$ are $J_{1}$ arbitrary martingale difference sequences.

(iii) The arbitrary processes can be interpreted as revision processes:

$$
\epsilon_{i}^{j}=E\left[y_{t+j} \mid I_{t}\right]-E\left[y_{t+j} \mid I_{t-1}\right] \quad j=H-J_{1}, \ldots, H-1
$$

It should be emphasized that equation (4.4) is a fully general reduced form of model (4.3). It does not involve any special assumption on the information set $I_{t}$ or any special form of the endogenous or exogenous variables (except that $u_{t}$ and $y_{t}$ are known at time $t$ ). Consequently, the information set may contain all kinds of extraneous variables. Their adjunction will simply increase the set of possible arbitrary martingale difference sequences (because the generated space is larger).

Moreover, the arbitrary processes need not be linear combinations of the variables included in the information set. This is a consequence of the fact that, in general, conditional expectations are not linear with respect to known variables. In the literature, some confusion between optimal forecasts and optimal linear forecasts is often present. For instance, Whiteman (1983) solves a general model involving optimal linear expectations. This framework is less general since it does not allow for totally unrestricted martingale difference sequences. Nevertheless, when one is concerned with linear stationary solutions (see Section 4.2) and when the innovation of the exogenous process is assumed to be independent, both approaches are equivalent. Furthermore, even in a stationary context, there exist nonlinear equilibria [see, e.g., formula (3.6)]. Using Whiteman's method, one cannot find these solutions on the basis of the information set $I_{t}=\left\{\epsilon_{t}, \epsilon_{t-1}, \ldots\right\}$.

To illustrate the results, consider, for instance, the following model:

$$
y_{t}=a E\left[y_{t+3} \mid I_{t-1}\right]+b E\left[y_{t+1} \mid I_{t-2}\right]+u_{t}
$$

It corresponds to $H=4(=\max \{t+3-(t-1), t+1-(t-2)\}), K=2$ (the expectations are formed at $t-1$ and $t-2$ ), and $J_{1}=3$ (the expected vari- 
ables are $y_{t+3}$ and $\left.y_{t+1}\right)$. Accordingly, three arbitrary martingale difference sequences enter the reduced form.

Table 5.2 gives the characterization of various models with their reduced forms ( $\epsilon^{j}$ being arbitrary martingale difference sequences).

The last example can readily be generalized to the class of models where all expectations are formed at time $t$ :

$$
y_{t}=\sum_{h=1}^{H} a_{h} E\left[y_{t+h} \mid I_{t}\right]+u_{t}
$$

We have $K=0$ and $J_{1}=H$. The reduced form is then

$y_{t}=\frac{-1}{a_{H}} \sum_{h=1}^{H-1} a_{H-h} y_{t-h}+\frac{1}{a_{H}} y_{t-H}+\sum_{h=1}^{H} a_{h} \sum_{j=0}^{h-1} \epsilon_{t+h-j-H}^{j}-\frac{1}{a_{H}} u_{t-H}$

where $\epsilon^{0}, \ldots, \epsilon^{H-1}$ are $H$ arbitrary martingale difference sequences.

The introduction of lagged terms in model (4.8) does not bring fundamental changes. If a sum $b_{1} y_{t-1}+\cdots+b_{K} y_{t-K}$ is added to the right-hand side of (4.6), the new lagged terms $\left(-1 / a_{H}\right)\left(b_{1} y_{t-H-1}+\cdots+b_{K} y_{t-H-K}\right)$ have to be added to the right-hand side of the reduced form. The order of the recursive equation changes from $H$ to $H+K$.

As shown above and illustrated in the examples, the multiplicity of the solutions derives from the value of $J_{1}$ which, in turn, is defined from the values of the structural coefficients $a_{k h}$. Consequently, models having a unique solution may directly be characterized. In fact, two cases can be considered: uniqueness up to initial conditions (no "specific" multiplicity) and "total" uniqueness (neither specific nor dynamic multiplicity).

In the first case, when $J_{1}=0$, only the dynamic multiplicity remains. The corresponding models do not contain expectations of future endogenous variables. A finite number of initial conditions is sufficient to select a unique solution. These models are given by

$$
y_{t}=\sum_{k=1}^{K} \sum_{1<k} a_{k 1} E\left[y_{t-1} \mid I_{t-k}\right]+\sum_{k=1}^{K} a_{k 0} y_{t-k}+u_{t}
$$

Such models are considered in Aoki and Canzoneri (1979), Visco (1981, 1984), and Broze and Szafarz (1984).

In the second case, when $J_{1}=J_{0}=0$, both causes of multiplicity (dynamics and martingale difference sequences) disappear. The corresponding models are, of course, very simple:

$$
y_{t}=\sum_{k=1}^{K} a_{k k} E\left[y_{t} \mid I_{t-k}\right]+u_{t}
$$

They are immediate generalizations of Muth's model. 
Table 5.2

\begin{tabular}{llllll}
\hline \hline $\begin{array}{l}\text { Model } \\
y_{t}=a E\left[y_{t} \mid I_{t-1}\right]+u_{t} \text { (Muth) }\end{array}$ & 1 & 1 & 0 & $y_{t}=\frac{a}{1-a} E\left[u_{t} \mid I_{t-1}\right]+u_{t}$ \\
$y_{t}=a E\left[y_{t+1} \mid I_{t}\right]+u_{t} \quad$ (Cagan) & 1 & 0 & 1 & $y_{t}=\frac{1}{a} y_{t-1}-\frac{1}{a} u_{t-1}+\epsilon_{t}^{0}$ \\
$y_{t}=a E\left[y_{t+1} \mid I_{t-1}\right]+u_{t}$ & 2 & 1 & 1 & $y_{t}=\frac{1}{a} y_{t-1}-\frac{1}{a} u_{t-1}+u_{t}-E\left[u_{t} \mid I_{t-1}\right]+\epsilon_{t-1}^{1}$ \\
$y_{t}=a E\left[y_{t+1} \mid I_{t}\right]+b y_{t-1}+u_{t}$ & 1 & 1 & 1 & $y_{t}=\frac{1}{a} y_{t-1}-\frac{b}{a} y_{t-2}-\frac{1}{a} u_{t-1}+\epsilon_{t}^{0}$ \\
$y_{t}=a E\left[y_{t+1} \mid I_{t-1}\right]+b E\left[y_{t} \mid I_{t-1}\right]+u_{t} \quad$ (Taylor) & 2 & 1 & 1 & $y_{t}=\frac{1-b}{a} y_{t-1}+u_{t}-E\left[u_{t} \mid I_{t-1}\right]+\frac{b-1}{a} u_{t-1}-\frac{b}{a} E\left[u_{t-1} \mid I_{t-2}\right]+\epsilon_{t-1}^{1}$ \\
$y_{t}=a\left[E y_{t+2} \mid I_{t}\right]+u_{t}$ & 2 & 0 & 2 & $y_{t}=\frac{1}{a} y_{t-2}-\frac{1}{a} u_{t-2}+\epsilon_{t}^{0}+\epsilon_{t-1}^{1}$ \\
$y_{t}=a E\left[y_{t+2} \mid I_{t}\right]+b E\left[y_{t+1} \mid I_{t}\right]+u_{t}$ & 2 & 0 & 2 & $y_{t}=-\frac{b}{a} y_{t-1}+\frac{1}{a} y_{t-2}-\frac{1}{a} u_{t-2}+\epsilon_{t}^{0}-\frac{b}{a} \epsilon_{t-1}^{0}+\epsilon_{t-1}^{1}$
\end{tabular}




\subsection{Linear stationary solutions}

As in Cagan's model, the transition from the general solution to the linear stationary solutions is greatly simplified by the use of the revision processes (Broze, Gourieroux, and Szafarz 1985a). Assume the following representations:

$$
\begin{aligned}
& u_{t}=A(B) \epsilon_{t}=\sum_{i=0}^{\infty} \alpha_{i} \epsilon_{t-i}, \quad \alpha_{0}=1 \\
& y_{t}=\Psi(B) \epsilon_{t}=\sum_{i=0}^{\infty} \psi_{i} \epsilon_{t-i}
\end{aligned}
$$

The corresponding revision processes are given by

$$
\epsilon_{i}^{j}=\psi_{j} \epsilon_{t} \quad j=H-J_{1}, \ldots, H-1
$$

Substituting in the reduced form and considering whether or not the characteristic polynomial $\Sigma_{i=0}^{J_{1}-J_{0}} a_{j_{1}-i}^{*} \lambda^{i}$ has explosive roots yields the following results:

(i) If $N$ denotes the number of roots of the characteristic polynomial that lie inside the unit circle, then if $J_{1}-N>0$, the general linear stationary solution involves $J_{1}-N$ free parameters; if $J_{1}-N=0$, there exists one linear stationary solution; and if $J_{1}-N<0$, there exist no linear stationary solutions.

(ii) When $J_{1}-N>0$, every arbitrary parameter $\psi_{j}$ is the coefficient of the regression of the revision process $\epsilon_{i}^{j}$ on the prediction error $u_{t}-E\left[u_{t} \mid I_{t-1}\right]$.

Some rational-expectations models may not admit a linear stationary solution. This is the case when the degree of freedom $\left(J_{1}\right)$ is smaller than the number of stationarity constraints $(N)$. For Cagan's model, this never happens. Consider, for instance, the fourth model given in Table 5.2:

$$
y_{t}=a E\left[y_{t+1} \mid I_{t}\right]+b y_{t-1}+u_{t}
$$

A model of this kind was studied by Sargan (1984). Its reduced form is

$$
y_{t}=\frac{1}{a} y_{t-1}-\frac{b}{a} y_{t-2}-\frac{1}{a} u_{t-1}+\epsilon_{t}^{0}
$$

where $\epsilon^{0}$ is an arbitrary martingale difference sequence $\left(J_{1}=1\right)$. Suppose for simplicity that the exogenous process is an independent white noise:

$$
u_{t}=\epsilon_{t}
$$

The general solution is then

$$
\left(a-B+b B^{2}\right) y_{t}=a \epsilon_{t}^{0}+\epsilon_{t-1}
$$


Table 5.3

\begin{tabular}{lccll}
\hline \hline$\lambda_{1}, \lambda_{2}$ & $N$ & $J_{1}-N$ & General linear stationary solution & Free parameter \\
\hline$\left|\lambda_{1}\right|>1,\left|\lambda_{2}\right|>1$ & 0 & 1 & $b\left(B-\lambda_{1}\right)\left(B-\lambda_{2}\right) y_{t}=\left(a \psi_{0}-B\right) \epsilon_{1}$ & $\downarrow_{0}$ \\
$\left|\lambda_{1}\right|>1,\left|\lambda_{2}\right| \leq 1$ & 1 & 0 & $b\left(B-\lambda_{1}\right) y_{t}=-\epsilon_{2}$ & - \\
$\left|\lambda_{1}\right| \leq 1,\left|\lambda_{2}\right| \leq 1$ & 2 & -1 & - & - \\
\hline \hline
\end{tabular}

The linear stationary solutions are such that

$$
\epsilon_{t}^{0}=\psi_{0} \epsilon_{t}
$$

Let $\lambda_{1}$ and $\lambda_{2}$ be the roots of the characteristic equation $a-B+b B^{2}=$ $b\left(B-\lambda_{1}\right)\left(B-\lambda_{2}\right)$. Three cases must be distinguished according to the relative positions of the modulus of $\lambda_{1}$ and $\lambda_{2}$ with respect to 1 . A synthetic view of the possibilities is given in Table 5.3. In the first case, the model has an infinity of linear stationary solutions with $\operatorname{ARMA}(2,1)$ representations and two $\mathrm{AR}(1)$ solutions corresponding to $\psi_{0}=\lambda_{2} / a$ and $\psi_{0}=\lambda_{1} / a$. In the second case, the model has a unique linear stationary solution with an AR(1) representation. In the last case, the model has no linear stationary solution.

\subsection{Perfect-foresight solutions}

Another way to solve the nonuniqueness problem is to select the perfectforesight solution of the model. This choice may seem less "ad hoc" than others, considering that the perfect foresight is the limiting case of rationality. To legitimize the argument, it is necessary to show that the solution of the perfect-foresight version of the model is always a special solution of its rational-expectations version. Unfortunately, this is not the case.

However it is possbble to characterize the models that will admit a perfect-foresight solution. They are characterized by $J_{1}=H$ (see Broze, Gourieroux, and Szafarz 1985a). If $J_{1}<H$, some (strong) additional conditions on the structure of the exogenous process are needed. If this is not the case, the solution of the perfect-foresight model will not verify the rational-expectations model.

Cagan's model $\left(J_{1}=H=1\right)$ belongs to the first category, but Taylor's model $\left(J_{1}=1 \neq H=2\right)$ and Muth's model $\left(J_{1}=0 \neq H=1\right)$ do not. In Section 2.6, it was shown that in the framework of Muth's model, perfect and rational expectations do coincide if and only if the exogenous variable $u_{t}$ is known at time $t-1$, that is, if it is predetermined. A white noise, for instance, does not fulfill this condition. Notice that this problem occurs also for many models having an infinity of solutions (see Table 5.2 
and compare the values of $H$ and $J_{1}$ ). Consider, for instance, Taylor's model (3.7):

$$
y_{t}=\frac{1}{\delta_{1}} E\left[y_{t+1} \mid I_{t-1}\right]-\frac{1}{\delta_{1}} E\left[y_{t} \mid I_{t-1}\right]-\frac{\delta_{0}}{\delta_{1}}-\frac{1}{\delta_{1}} \epsilon_{t}
$$

where $\epsilon$ is an independent white noise. Taking the rational expectation given $I_{t-1}$ yields

$$
E\left[y_{t} \mid I_{t-1}\right]=\frac{1}{\delta_{1}} E\left[y_{t+1} \mid I_{t-1}\right]-\frac{1}{\delta_{1}} E\left[y_{t} \mid I_{t-1}\right]-\frac{\delta_{0}}{\delta_{1}}
$$

The difference of the last two equations, that is, the difference between perfect and rational expectations, is

$$
y_{t}-E\left[y_{t} \mid I_{t-1}\right]=\epsilon_{t}
$$

Taylor's model has no perfect-foresight solution except when it does not include a disturbance term.

Another question related to the perfect-foresight versus rational-expectations models comes naturally: Are these models observationally distinguishable? We will analyze this problem for Muth's simpler model, assuming that the exogenous process has an autoregressive structure of order 1:

$$
\begin{aligned}
& y_{t}=a \hat{y}_{t \mid t-1}+x_{t} b+v_{t} \\
& x_{t}=\rho x_{t-1}+w_{t} \quad|\rho|<1
\end{aligned}
$$

We adopt the usual assumptions about the independence of $v$ and $w$ and denote by $\sigma_{v}^{2}$ and $\sigma_{w}^{2}$ their respective variances.

Under the rational-expectations hypothesis $\left(\hat{y}_{t \mid t-1}=E\left[y_{t} \mid I_{t-1}\right]\right)$, the reduced form of $(4.12)$ is

$$
\begin{aligned}
& y_{t}=\frac{b \rho}{1-a} x_{t-1}+w_{t} b+v_{t} \\
& x_{t}=\rho x_{t-1}+w_{t}
\end{aligned}
$$

The identification at the first order gives $b \rho /(1-a)$ and $\rho$. The identification at the second order adds $\sigma_{w}^{2}, \sigma_{v}^{2}+b^{2} \sigma_{w}^{2}$, and $b \sigma_{w}^{2}$. It follows that at the first two orders, the rational-expectation model is just identified.

Consider now the perfect-foresight model obtained by setting $\hat{y}_{t \mid t-1}=$ $y_{1}$. Its reduced form is

$$
\begin{aligned}
& y_{t}=\frac{b \rho}{1-a} x_{t-1}+\frac{b}{1-a} w_{t}+\frac{1}{1-a} v_{t} \\
& x_{t}=\rho x_{t-1}+w_{t}
\end{aligned}
$$


The identification at the first order gives $b \rho /(1-a)$ and $\rho$. At this level, it is not possible to distinguish the perfect-foresight from the rationalexpectations model. However, identification at the second order gives only $\sigma_{w}^{2}$ and $[b /(1-a)]^{2} \sigma_{w}^{2}+[1 /(1-a)]^{2} \sigma_{v}^{2}$ since $[b /(1-a)] \sigma_{w}^{2}$ does not add anything. The model is not identifiable. Consequently, for $a \neq 0$, the transition from perfect foresight to rational expectations makes the model identifiable. This result is established in Gourieroux, Monfort, and Renault (1985) as a preliminary for test procedures.

\section{Multivariate models}

Multivariate linear rational-expectations models were considered by several authors (Wallis 1980; Pesaran 1981; Wegge 1984). The solution techniques used for unidimensional models can be easily generalized provided that invertibility conditions are imposed on the matrices multiplying the expectations vector.

Consider, for instance, the model

$$
y_{t}=\pi E\left[y_{t+1} \mid I_{t}\right]+u_{t}
$$

where the endogenous vector $y_{t}$ has $G$ components, the vector $u_{t}$ summarizing all exogenous and disturbance terms has also $G$ components, and the matrix $\pi$ is of size $G \times G$. This model is an extension of Cagan's model.

Let us first suppose that $\pi$ is nonsingular. The martingale solution technique can be used. The general solution is then given by

$$
y_{t}=y_{t}^{0 *}+\pi^{-t} M_{t}
$$

where $y_{t}^{0 *}$ is a particular solution (e.g., backward or forward), and $M$ is a $G$-dimensional vector with arbitrary martingale components.

The solution technique based on martingale difference sequences can also be applied. It gives the following reduced form:

$$
y_{t}=\pi^{-1} y_{t-1}-\pi^{-1} u_{t-1}+\epsilon_{t}
$$

where $\epsilon$ is a $G$-dimensional vector with arbitrary martingale difference sequence components.

The assumption that $\pi$ is invertible is quite strong. In many cases, $\pi$ has a large number of zero elements: Some expectations appear as explanatory variables for an endogenous variable whereas others have no a priori influence on this variable. Technically, if $\pi$ is singular, new difficulties emerge. Consider, for instance, the case where $\pi$ is a nilpotent matrix of index $2: \pi^{2}=0$. In this case, the solution is unique, 


$$
\begin{aligned}
y_{t} & =\pi E\left[y_{t+1} \mid I_{t}\right]+u_{t} \\
& =\pi E\left[\pi E\left[y_{t+2} \mid I_{t+1}\right]+u_{t+1} \mid I_{t}\right]+u_{t} \\
& =\pi^{2} E\left[y_{t+2} \mid I_{t}\right]+\pi E\left[u_{t+1} \mid I_{t}\right]+u_{t} \\
& =\pi E\left[u_{t+1} \mid I_{t}\right]+u_{t}
\end{aligned}
$$

and a forward-looking approach is used to obtain the uniqueness result. The recursive substitutions are always valid since matrix $\pi$ need not be inverted. Therefore, irrespective of matrix $\pi$, fixing a terminal period $T$ allows for the formulation

$$
y_{t}=\pi^{T-t} E\left[y_{T} \mid I_{t}\right]+\sum_{i=0}^{T-t-i} \pi^{i} E\left[u_{t+i} \mid I_{t}\right] \quad t<T
$$

With a terminal condition on $E\left[y_{t} \mid I_{t}\right]$, it is possible to obtain a particular solution of the model. This approach was suggested by Chow (1980) for the univariate case and by Wegge (1984) for the multivarate case.

Looking for a general solution valid for any matrix $\pi$, we will isolate a submodel of (5.1) in which a nonsingular matrix multiplies the expectations vector. Two methods will be suggested. Each one leads to a canonical form of model (5.1).

Theorem 5.1. The G-dimensional process y may be decomposed into two subprocesses $y^{1}$ and $y^{2}$, of respective dimensions $G_{1}$ and $G_{2}=G-G_{1}$, in such a way that model (5.1) is equivalent to

$y_{t}^{1}=C_{1} E\left[y_{t+1}^{1} \mid I_{t}\right]+w_{t}^{1} \quad y_{t}^{2}=C_{2} y_{t}^{1}+w_{t}^{2}$

where $C_{1}$ is a $G_{1} \times G_{1}$ nonsingular matrix and $w_{t}^{1}$ and $w_{t}^{2}$ are functions of the exogenous variables and their expectations.

Proof. See Appendix C.

The canonical form given by Theorem (5.1) is obviously not unique if only because of the various possible choices of $y^{1}$.

Notice that the $G_{1}$ endogenous variables $y_{t}^{1}$ are determined in the first block and the $G_{2}$ variables $y_{t}^{2}$ are obtained, knowing $y_{t}^{1}$, by the last equations.

The second canonical form uses the Jordan form of matrix $\pi$. Suppose that $Q$ is an invertible matrix such that $Q \pi Q^{-1}$ is the Jordan form of $\pi$ :

$$
Q \pi Q^{-1}=\left(\begin{array}{cc}
P & 0 \\
0 & N
\end{array}\right)
$$

where $N$ is the block associated with the zero eigenvalue. The canonical form is expressed using the vectors 


$$
y_{i}^{*}=Q y_{t}=\left[\begin{array}{l}
y_{i}^{* 1} \\
y_{i}^{* 2}
\end{array}\right] \quad u_{i}^{*}=Q u_{t}=\left[\begin{array}{l}
u_{i}^{* 1} \\
u_{i}^{* 2}
\end{array}\right]
$$

The splitting of $y^{*}$ and $u^{*}$ is made following the block partition of the Jordan form.

Theorem 5.2. Model (5.1) is equivalent to

$y_{i}^{* 1}=P E\left[y_{i+1}^{* 1} \mid I_{t}\right]+u_{i}^{* 1} \quad y_{i}^{* 2}=N E\left[y_{i+1}^{* 2} \mid I_{t}\right]+u_{i}^{* 2}$

where $P$ is a $K_{1}$-square nonsingular matrix and $N$ is a $K_{2}$-square nilpotent matrix.

The order of $N$, that is, $K_{2}$, is equal to the number of zero eigenvalues of $\pi$. In this canonical form, the two blocks are totally separated. Each group of variables cannot be influenced by expectations of variables in the other group.

In both canonical forms, the first group of equations reduces to a system formally equivalent to

$$
y_{t}^{1}=\alpha E\left[y_{t+1}^{1} \mid I_{t}\right]+v_{t}
$$

where $\alpha$ is a nonsingular matrix. It was shown in the beginning of the section that such a model can be solved without any difficulty through either a martingale or a martingale difference sequence method. We will consider hereafter the second method. While the general solution of model (5.1) may be deduced from any of the canonical forms, comparative results will be used to facilitate the study of the multiplicity problem.

Theorem 5.3. The solutions of model (5.1) are given by

$y_{t}^{1}=C_{1}^{-1} y_{t-1}^{1}-C_{1}^{-1} w_{t-1}^{1}+\epsilon_{t}^{1} \quad y_{t}^{2}=C_{2} y_{t}^{1}+w_{t}^{2}$

where $\epsilon^{1}$ is a vector with $G_{1}$ arbitrary martingale difference sequences components.

Proof. The proof is straightforward from Theorem (5.1) since $C_{1}$ is invertible.

Theorem 5.4. The solutions of model (5.1) are given by

$y_{t}=Q^{-1} y_{i}^{*}$

where $y^{*}$ verifies

$y_{i}^{* 1}=P^{-1} y_{i-1}^{* 1}-P^{-1} u_{i-1}^{* 1}+\epsilon_{i}^{* 1}$

$y_{i}^{* 2}=\sum_{k=0}^{K_{2}-1} N^{k} E\left[u_{i+k}^{* 2} \mid I_{t}\right]$ 
and $\epsilon^{* 1}$ is a vector with $K_{1}$ arbitrary martingale difference sequences components.

Proof.

(a) The reduction of the first group of equations is straightforward from Theorem 5.2 since $P$ is invertible.

(b) In the second group of equations, there is a nilpotent matrix $N$ : $N^{K_{2}}=0$. A forward-looking approach gives a unique solution:

$$
\begin{aligned}
y_{i}^{* 2} & =N E\left[y_{i+1}^{* 2} \mid I_{t}\right]+u_{i}^{* 2} \\
& =N^{2} E\left[y_{i+2}^{* 2} \mid I_{t}\right]+N E\left[u_{i+1}^{* 2} \mid I_{t}\right]+u_{i}^{* 2} \\
& \vdots \\
& =N^{K_{2}} E\left[y_{i+K_{2}}^{* 2} \mid I_{t}\right]+N^{K_{2}-1} E\left[u_{i+K_{2}-1}^{* 2} \mid I_{t}\right]+\cdots+u_{i}^{* 2} \\
& =\sum_{k=0}^{K_{2}-1} N^{k} E\left[u_{i+k}^{* 2} \mid I_{t}\right]
\end{aligned}
$$

We have now two generally different expressions for the set of solutions to model (5.1). The first comes out an iterative procedure. It is useful to obtain an explicit form of the solutions. The second describes the multiplicity of solutions.

The two solutions are, of course, equivalent. In particular, they involve the same number of arbitrary martingale difference sequences. The one-to-one link between $\epsilon^{1}$ and $\epsilon^{* 1}$ can be made explicit as follows. The vector $y^{*}$ was previously defined as

$$
y_{i}^{*}=Q y_{t}=Q R\left[\begin{array}{l}
y_{t}^{1} \\
y_{t}^{2}
\end{array}\right]=\bar{Q}\left[\begin{array}{l}
y_{t}^{1} \\
y_{t}^{2}
\end{array}\right]
$$

where $R$ is the matrix that takes into account the possible permutations among the components of $y_{t}$. It follows that

$$
y_{t}^{* 1}=\bar{Q}_{11} y_{i}^{1}+\bar{Q}_{12} y_{t}^{2}
$$

and the prediction error associated with $y_{i}^{* 1}$ is

$$
\epsilon_{t}^{* 1}=\left(\tilde{Q}_{11}+\bar{Q}_{12} C_{2}\right) \epsilon_{t}^{1}+\bar{Q}_{12}\left(w_{t}^{2}-E\left[w_{t}^{2} \mid I_{t-1}\right]\right)
$$

Conversely,

$$
\left[\begin{array}{l}
y_{i}^{1} \\
y_{i}^{2}
\end{array}\right]=\bar{Q}^{-1} y_{i}^{*}
$$

and

$$
y_{t}^{1}=\left(\tilde{Q}^{-1}\right)_{11} y_{t}^{* 1}+\left(\tilde{Q}^{-1}\right)_{12} y_{i}^{* 2}
$$

Consequently, 
$\epsilon_{i}^{1}=\left(\bar{Q}^{-1}\right)_{11} \epsilon_{i}^{* 1}+\left(\bar{Q}^{-1}\right)_{12} \sum_{k=0}^{K_{2}-1} N^{k}\left(E\left[u_{t+k}^{* 2} \mid I_{t}\right]-E\left[u_{t+k}^{* 2} \mid I_{t-1}\right]\right)$

Equations (5.13) and (5.14) exhibit the equivalence between $\epsilon^{1}$ and $\epsilon^{* 1}$. It follows that $G_{1}=K_{1}$ and that in both expressions of the general solution, the number of arbitrary martingale difference sequences is equal to $K_{1}$, the number of nonzero eigenvalues of matrix $\pi$.

Two extreme cases can be mentioned. First, when $\pi$ is nonsingular $\left(K_{1}=G\right), G$ arbitrary martingale difference sequences enter the general solution. Second, when $\pi$ is nilpotent $\left(K_{1}=0\right)$, the solution is unique:

$$
y_{t}=\sum_{k=0}^{G-1} \pi^{k} E\left[u_{t+k} \mid I_{t}\right]
$$

In particular, if $\pi$ is a lower strictly triangular matrix, the system is recursive with respect to the expectations,

$$
\begin{aligned}
& y_{t}(1)=v_{t}(1) \\
& y_{t}(2)=\pi(2,1) E\left[y_{t+1}(1) \mid I_{t}\right]+v_{t}(2) \\
& y_{t}(3)=\pi(3,1) E\left[y_{t+1}(1) \mid I_{t}\right]+\pi(3,2) E\left[y_{t+1}(2) \mid I_{t}\right]+v_{t}(3)
\end{aligned}
$$

and the unique solution may be directly computed by recursive substitutions.

\section{6}

\section{Conclusion}

The analysis of the solutions of univariate linear rational-expectations models is now quite advanced. For any model of this type, it is possible to describe the set of all solutions. Also linear stationary solutions are parameterized in a simple way: The number of free parameters is immediately deduced from the values of the structural coefficients of the model. The confusion between rational expectations and perfect foresight found in some papers is clarified.

Up to now, few authors have considered multivariate rational-expectations models. These models offer, however, a promising ground for further investigation. The results of Section 5 show that the multivariate case cannot be treated as a simple generalization of the univariate case. For instance, a unique solution may occur in models including expectations of future endogenous variables.

Another extension concerns nonlinear rational-expectations models. For these models, a numerical solution technique is given by Fair and Taylor (1983). Grossman and Stiglitz (1980) and Hellwig (1982) consider a mean-variance model including rational expectations. In fact, the method 
based on revision processes also applies to models of this type (Broze, Gourieroux, and Szafarz 1985b).

Finally, the econometric problems linked to the identification and estimation of rational-expectations models are probably those that most urgently need a solution. Important results in this field have been proposed by Wallis (1980), Pesaran (1981), Hansen and Sargent (1980, 1982), Hansen and Singleton (1982), and Wickens (1982). They open the way to further developments that should lead to practical applications.

\section{Appendix A: Proof of Theorem 3.1}

Lemma. The solution of $p_{i}^{0}$ of (3.9) has minimum variance in the set of all solutions if and only if

$$
\operatorname{cov}\left(p_{t}^{0}, p_{i}^{*}\right)=0
$$

for any $t$ and for any solution $p_{i}^{*}$ of the homogeneous equation

$$
p_{t}=a E\left[p_{t+1} \mid I_{t}\right]
$$

Proof. Gourieroux et al. (1982, p. 410) show that the general solution of (3.9) is

$$
p_{t}=p_{t}^{0}+p_{i}^{*}
$$

where $p_{t}^{0}$ is a particular solution of (3.9) and $p_{t}^{*}=\left(1 / a^{t}\right) M_{t}$ is the general solution of (A.2). $M$ refers to an arbitrary martingale.

The set of solutions $p_{i}^{*}$ of (A.2) is a vector space. Therefore, a minimum-variance solution $p_{t}^{0}$ must fulfill

$$
V\left(p_{t}^{0}\right) \leq V\left(p_{i}^{0}+\lambda p_{i}^{*}\right) \quad \forall \lambda, \forall t
$$

This condition is equivalent to

$$
\lambda^{2} V\left(p_{i}^{*}\right)+2 \lambda \operatorname{cov}\left(p_{i}^{0}, p_{i}^{*}\right) \geq 0, \quad \forall \lambda, \forall t
$$

The last inequality occurs for any value of $\lambda$ only if

$$
\operatorname{cov}\left(p_{t}^{0}, p_{i}^{*}\right)=0 \quad \forall t
$$

Finally, condition (A.1) is necessary and sufficient since all the conditions mentioned above are equivalent.

Proof of Theorem 3.1. Let us fix a date $t_{0}$ and consider the value $p_{t_{0}}^{0}$ at $t_{0}$ of a potential minimum-variance solution. Using the lemma, we could write

$$
\operatorname{cov}\left(p_{i}^{0}, p_{i}^{*}\right)=\operatorname{cov}\left(p_{t}^{0}, \frac{1}{a^{t}} M_{t}\right)=\frac{1}{a^{t}} \operatorname{cov}\left(p_{t}^{0}, M_{t}\right)=0
$$


In particular, if we choose the martingale

$$
M_{i}^{0}= \begin{cases}p_{t_{0}}^{0} & t=t_{0} \\ E\left[p_{t_{0}}^{0} \mid I_{t}\right] & t<t_{0} \\ p_{t_{0}}^{0}+\Sigma_{i=t_{0}+1}^{t} \epsilon_{i} & t>t_{0}\end{cases}
$$

condition (A.3) would then imply for $t=t_{0}$ that

$$
\operatorname{cov}\left(p_{t_{0}}^{0}, M_{t_{0}}^{0}\right)=V\left(p_{t_{0}}^{0}\right)=0
$$

and therefore variable $p_{t_{0}}^{0}$ would be deterministic. Since the previous argument holds at any time, it follows that $p_{i}^{0}$ would be a deterministic function of $t$. However, by substituting such a function $f(t)$ for $p_{t}$ in model (2.1), we obtain

$$
f(t)=a f(t+1)+u_{t}
$$

which is impossible since

$$
V\left(u_{t}\right)=V\left(\sum_{i=0}^{\infty} \theta_{i} \epsilon_{t-i}\right)=\sigma^{2} \sum_{i=0}^{\infty} \theta_{i}^{2} \neq 0
$$

\section{Appendix B: Proof of Theorem 3.2}

We first introduce the theoretical linear regression of $p_{t}$ on $u_{t}, u_{t-1}, \ldots$, denoted by $E L\left[p_{t} \mid I_{t}\right]$.

Lemma. If $p_{t}$ is a solution of (3.9), the optimal linear expectation EL $\left[p_{t} \mid I_{t}\right]$ is also a solution of (3.9).

Proof. We apply the operator $E L\left[\cdot \mid I_{t}\right]$ to both sides of (3.9):

$$
\begin{aligned}
E L\left[p_{t} \mid I_{t}\right] & =a E L\left[E\left[p_{t+1} \mid I_{t}\right] \mid I_{t}\right]+E L\left[u_{t} \mid I_{t}\right] \\
& =a E L\left[p_{t+1} \mid I_{t}\right]+u_{t} \\
& =a E L\left[E L\left[p_{t+1} \mid I_{t+1}\right] \mid I_{t}\right]+u_{t}
\end{aligned}
$$

Since $E L\left[p_{t+1} \mid I_{t+1}\right]$ is a linear function of $u_{t+1}, u_{t}, \ldots$, and $\left(u_{t}\right)$ is a moving-average process, we have

$$
E L\left[E L\left[p_{t+1} \mid I_{t+1}\right] \mid I_{t}\right]=E\left[E L\left[p_{t+1} \mid I_{t+1}\right] \mid I_{t}\right]
$$

and replacing in (B.1), we obtain the result.

Proof of Theorem (3.2). Using the inequality

$$
V\left(p_{t}\right) \geq V\left(E L\left[p_{t} \mid I_{t}\right]\right)
$$

and the lemma, we see that the variance of any stationary solution is always larger than the variance of a linear stationary solution. Therefore, 
the minimum-variance stationary solution is in the subset of linear solutions.

\section{Appendix C: Proof of Theorem 5.1}

First, if $\pi$ is nonsingular, Theorem 5.1 is straightforward with $G_{1}=G$, $C_{1}=\pi, y_{t}^{1}=y_{t}$, and $w_{t}^{1}=u_{t}$.

Consider the case where $\pi$ is singular. Permuting, if necessary, the components of $y$, it is possible to partition $\pi$ into

$$
\pi=\left(\begin{array}{ll}
\pi_{11} & \pi_{12} \\
\pi_{21} & \pi_{22}
\end{array}\right)
$$

where $\pi_{11}, \pi_{12}, \pi_{21}$, and $\pi_{22}$ are (respectively) of sizes $r \times r, r \times(G-r)$, $(G-r) \times r$, and $(G-r) \times(G-r), r$ denoting the rank of $\pi$ and the rank of $\left(\pi_{11}, \pi_{12}\right)$.

The $G-r$ last rows are linear combinations of the $r$ first rows. Consequently, there exists a $(G-r) \times r$ matrix $\Lambda$ such that

$$
\pi_{21}=\Lambda \pi_{11} \text { and } \pi_{22}=\Lambda \pi_{12}
$$

Model (5.1) then becomes

$$
\begin{aligned}
& y_{t}^{1}=\pi_{11} E\left[y_{t+1}^{1} \mid I_{t}\right]+\pi_{12} E\left[y_{t+1}^{2} \mid I_{t}\right]+u_{t}^{1} \\
& y_{t}^{2}=\Lambda \pi_{11} E\left[y_{t+1}^{1} \mid I_{t}\right]+\Lambda \pi_{12} E\left[y_{t+1}^{2} \mid I_{t}\right]+u_{t}^{2}
\end{aligned}
$$

or, equivalently,

$$
\begin{aligned}
& y_{t}^{1}=\pi_{11} E\left[y_{t+1}^{1} \mid I_{t}\right]+\pi_{12} E\left[y_{t+1}^{2} \mid I_{t}\right]+u_{t}^{1} \\
& y_{t}^{2}=\Lambda\left(y_{t}^{1}-u_{t}^{1}\right)+u_{t}^{2}
\end{aligned}
$$

Advancing the time subscripts of the second equation by 1 and taking the conditional expectation on $I_{t}$ yields

$$
E\left[y_{t+1}^{2} \mid I_{t}\right]=\Lambda\left(E\left[y_{t+1}^{1} \mid I_{t}\right]-E\left[u_{t+1}^{1} \mid I_{t}\right]\right)+E\left[u_{t+1}^{2} \mid I_{t}\right]
$$

Using (C.2) to substitute for $E\left[y_{t+1}^{2} \mid I_{t}\right]$ in the first equation of (C.1) gives $y_{t}^{1}=\left(\pi_{11}+\pi_{12} \Lambda\right) E\left[y_{t+1}^{1} \mid I_{t}\right]+u_{t}^{1}+\pi_{12} E\left[u_{t+1}^{2} \mid I_{t}\right]-\pi_{12} \Lambda E\left[u_{t+1}^{1} \mid I_{t}\right]$ $y_{t}^{2}=\Lambda y_{t}^{1}+u_{t}^{2}-\Lambda u_{i}^{1}$

(a) If $\left(\pi_{11}+\pi_{12} \Lambda\right)$ is nonsingular, the theorem is proved.

(b) If $\left(\pi_{11}+\pi_{12} \Lambda\right)$ is singular, it is possible to start the decomposition procedure anew with the first group of equations in (C.3). Each step reduces the size of $y^{1}$. At most, $G$ steps are thus needed to conclude.

Finally, the matrix that multiplies the expectations vector is nonsingular. It is denoted $C_{1}$. 


\section{References}

Aoki, M., and M. Canzoneri (1979), "Reduced Forms of Rational Expectations Models," Quarterly Journal of Economics, 93, 59-71.

Blanchard, O. J. (1979), "Backward and Forward Solutions for Economies with Rational Expectations," American Economic Review, 69, 114-18.

Blanchard, O. J., and C. M. Kahn (1980), "The Solution of Linear Difference Models under Rational Expectations," Econometrica, 48, 1305-11.

Bray, M. (1981), "Future Trading, Rational Expectations and the Efficient Markets Hypothesis," Econometrica, 49, 575-96.

Bray, M., and N. Savin (1984), "Rational Expectations Equilibria: Learning and Models Specification," Discussion Paper 79, University of Cambridge.

Broze, L., C. Gourieroux, and A. Szafarz (1985a), "Solutions of Dynamic Linear Rational Expectations Models," Econometric Theory, 1, 341-68.

(1985b), "Equilibrium Asset Prices in a Dynamic Mean-Variance Model with Rational Expectations," working paper, Université Libre de Bruxelles.

Broze, L., and A. Szafarz (1984), "On Linear Models with Rational Expectations which Admit a Unique Solution," European Economic Review, 24, 103-11. (1985), "Forme Réduite d'un Modèle Général à Anticipations Rationnelles," Cahiers du CERO, 2, 175-205.

Cagan, P. (1956), "The Monetary Dynamics of Hyperinflation," in Studies in the Quantity Theory of Money, M. Friedman (ed.), University of Chicago Press, Chicago, IL.

Chow, G. C. (1980), "Econometric Policy Evaluation and Optimization under Rational Expectations," Journal of Economic Dynamics and Control, 2, 4759.

Evans, G., and S. Honkapohja (1986), "A Complete Characterization of ARMA Solutions to Linear Rational Expectations Models," Review of Economic Studies, 53, 227-39.

Fair, R. C., and J. B. Taylor (1983), "Solution and Maximum Likelihood Estimation of Dynamic Non-linear Rational Expectations Models," Econometrica, 51, 1169-84.

Fourgeaud, C., C. Gourieroux, and J. Pradel (1986), "Learning Procedure and Convergence to Rationality," Econometrica, 54, 845-68.

Gourieroux, C., J. J. Laffont, and A. Monfort (1982), "Rational Expectations in Dynamic Linear Models: Analysis of the Solutions," Econometrica, 50, 40925 .

Gourieroux, C., A. Monfort, and E. Renault (1985), "Testing Unknown Linear Restrictions on Parameter Functions," Discussion Paper CEPREMAP No. 8516.

Grossman, S. J., and J. E. Stiglitz (1980), "On the Impossibility of Informationally Efficient Markets," American Economic Review, 70, 393-408.

Hansen, L. P., and T. J. Sargent (1980), "Formulating and Estimating Linear Rational Expectations Models," Journal of Economic Dynamics and Control, 2, 7-46.

(1982), "Instrumental Variables Procedures for Estimating Linear Rational Expectations Models," Journal of Monetary Economics, 9, 263-96.

Hansen, L. P., and K. J. Singleton (1982), "Generalized Instrumental Variables Estimation of Non-Linear Rational Expectations Models," Econometrica, 50, 1269-86. 
Hellwig, M. F. (1982), "Rational Expectations Equilibrium with Conditioning on Past Prices: A Mean-Variance Example," Journal of Economic Theory, 26, 279-312.

Lucas, R. E. (1976), "Econometric Policy Evaluation: A Critique," in The Phillips Curve and Labor Market, K. Brunner and A. M. Meltzer (eds.), NorthHolland, Amsterdam.

McCallum, B. T. (1976), "Rational Expectations and the Estimation of Econometric Models: An Alternative Procedure," International Economic Review, 17, 484-90.

(1983), "On Non-Uniqueness in Rational Expectations Models: An Attempt at Perspective," Journal of Monetary Economics, 11, 139-68.

Muth, J. F. (1961), "Rational Expectations and the Theory of Price Movements," Econometrica, 29, 315-35.

Nerlove, M. (1958), "Adaptive Expectations and Cobweb Phenomena," Quarterly Journal of Economics, 73, 227-40.

Pesaran, M. H. (1981), "Identification of Rational Expectations Models," Journal of Econometrics, 16, 375-98.

Sargan, J. D. (1984), "Alternative Models for Rational Expectations in Some Simple Irregular Cases," Discussion Paper, London School of Economics.

Sargent, T. J., and N. Wallace (1975), "Rational Expectations, the Optimal Monetary Instruments and the Optimal Money Supply Rule," Journal of Political Economy, 83, 241-55.

(1976), "Rational Expectations and the Theory of Economic Policy," Journal of Monetary Economics, 2, 169-84.

Shiller, R. J. (1978), "Rational Expectations and the Dynamic Structure of Macroeconomic Models: A Critical Review," Journal of Monetary Economics, 4, $1-44$.

Taylor, J. B. (1977), "Conditions for Unique Solutions in Stochastic Macroeconomic Models with Rational Expectations," Econometrica, 45, 1377-85.

(1979), "Estimation and Control of a Macroeconomic Model with Rational Expectations," Econometrica, 47, 1267-86.

Vicso, I. (1981), "On the Derivation of Reduced Forms of Rational Expectations Models," European Economic Review, 16, 355-65.

(1984), "On Linear Models with Rational Expectations: An Addendum," European Economic Review, 24, 113-15.

Wallis, K. F. (1980), "Econometric Implications of the Rational Expectations Hypothesis," Econometrica, 48, 49-73.

Watson, M. (1985), "Recursive Solution Methods for Dynamic Linear Rational Expectations Models," Discussion Paper, Harvard University.

Wegge, L. L. (1984), "Identifiability of Structural Models Containing Muth-Rational Current and Future Expectations," Working Paper 233, University of California.

Whiteman, C. H. (1983), Linear Rational Expectations Models: A User's Guide, University of Minnesota Press, Minneapolis, MN.

(1985), "Analytical Policy Design Under Rational Expectations," mimeo, University of Iowa.

Wickens, M. R. (1982), "The Efficient Estimation of Econometric Models with Rational Expectations," Review of Economic Studies, 44, 55-67. 